\section{PROSPECTIVA Y CAMBIO SOCIAL: ¿CÓMO ORIENTAR LAS POLÍTICAS DE INVESTIGACIÓN Y DESARROLLO EN LAS SOCIEDADES TECNOLÓGICAS AVANZADAS?}

\author{
Antonio López Peláez \\ Departamento de Sociología III \\ Universidad Nacional de \\ Educación a Distancia-UNED
}

\section{PROSPECTIVE AND SOCIAL CHANGE: ¿HOW TO DIRECT THE RESEARCH AND INNOVATION POLICIES IN THE TECHNOLOGICAL ADVANCED SOCIETIES?}

\begin{abstract}
The analysis of the future and taking decisions related to the tendencies of the detectable technological and social change in our present time has turned into a basic tool in the scientific-technological policy of the contemporary societies. The technological development can not be improvised: technologies have a long gestation period and public policies about science and technology should establish some financing guidelines that make possible to develop a technological path which may be considered as strategic. At the same time, the technological advances generate different kind of risks and the citizens who live in advanced democratic societies claim to participate in the scientific-technological policy, not only through their legitimate political representatives but also through their own behaviour like consumers. In this article, the basic dimensions of the denominated Futures Studies are analyzed, the most outstanding contributions and the actual situation of the prospective investigations in Spain and The European Union.
\end{abstract}

KEY WORDS: Prospective, future studies, strategic planning, social change and social trends.

\section{Introducción}

Las políticas de I+D e innovación son políticas estratégicas en las sociedades contemporáneas. Se establecen tomando en consideración, entre otras variables, las previsiones sobre los avances tecnológicos previsibles, su impacto socioeconómico, y la posición que se busca ocupar en ese futuro a corto, medio y largo plazo. En este artículo presentamos los rasgos básicos de lo que podemos denominar Prospectiva o Estudios sobre el Futuro (Future Studies). Analizaremos sus caracteristicas, las instituciones que se han creado para
RESUMEN: El análisis del futuro, y la toma de decisiones en función de las tendencias de cambio tecnológico y social detectables en el presente, se han convertido en una herramienta básica en la política científico-tecnológica de las sociedades contemporáneas. El desarrollo tecnológico no se improvisa: las tecnologias tienen un largo período de gestación, y las politicas públicas sobre la ciencia y la tecnología deben establecer pautas de financiación que hacen posible desarrollar unas trayectorias tecnológicas consideradas estratégicas. A la vez, los avances tecnológicos generan riesgos de diverso tipo, y los ciudadanos, en sociedades democráticas avanzadas, reclaman participar en el diseño de la política científico-tecnológi$\mathrm{ca}$, tanto a través de sus legitimos representantes políticos, como a través de su propio comportamiento como consumidores. En este artículo, se analizan las dimensiones básicas de los denominados Future Studies, las aportaciones más destacables, y la situación actual de las investigaciones prospectivas en España y la Unión Europea.

PALABRAS CLAVE: Prospectiva, estudios de futuro, planificación estratégica, cambio social, tendencias sociales.

desarrollarlos, y el papel crucial que juegan en nuestro sistema de I+D e innovación, al aportar información cualificada para la toma de decisiones.

El futuro siempre ha sido un motivo de preocupación para los seres humanos. Podemos rastrear a lo largo de la historia las diversas formas de analizar las tendencias de cambio inscritas en el presente, y cómo anticipan las caracteristicas del futuro. Desde los oráculos en la tradición clásica (Hernández de la Fuente, 2008), hasta los modernos estudios prospectivos (Fowles, 1978; EFILWC, 2003; 
Georghiou, Harper, Keenan, Miles, Popper, 2008), la acción en el presente no se entiende sin tomar en consideración el objetivo perseguido que se quiere alcanzar en un plazo de tiempo determinado.

La diferencia básica entre las distintas formas de previsión a lo largo de la historia reside precisamente en la noción de futuro con la que operan. En la antigüedad, y hasta nuestros días, la tradición del determinismo (tecnológico, histórico, social) postula una lógica única para la evolución de los acontecimientos, en la que los sujetos humanos son receptores pasivos del cambio, y sólo pueden protagonizarlo en la medida en que dicho proceso les favorece (de ahí los diversos ritos para sondear si los dioses están de nuestra parte). Este modelo llega hasta el análisis heideggeriano de la esencia de la técnica, lo que denomina das Gestell, en él no queda sino esperar un nuevo advenimiento (Heidegger, 1975). En este planteamiento extremo, también el futuro se determina por una ley interna, ajena a nosotros, llevando el determinismo tecnológico a su máxima expresión.

Esta conceptualización del futuro desde la óptica del determinismo entra en crisis tras la experiencia de la Segunda Guerra Mundial, y el desarrollo de la denominada Big Science. El futuro aparece ahora como un campo de juego para la acción humana: no se puede esperar pasivamente, se conquista, y por lo tanto necesitamos métodos y técnicas para analizarlo y construirlo en función de nuestros intereses políticos, económicos o militares. Objetivos tecnológicos como la bomba atómica requieren años de planificación y esfuerzo conjunto, aportación de cuantiosos recursos, y una evaluación clara de los beneficios (que se derivan no sólo de conseguir una tecnología clave en el ámbito militar o en el ámbito civil, sino también de la evaluación del coste o peligro que supone que otros países o empresas la obtengan antes y la desarrollen mejor).

En este nuevo contexto, la prospectiva tecnológica se convierte en una herramienta estratégica, y pasa a considerarse un elemento clave en los programas de I+D e innovación de los paises tecnológicamente avanzados. En un entorno de incertidumbre, fuerte competencia entre empresas y paises, y ante la necesidad de invertir recursos económicos y humanos (que siempre son limitados) en tecnologias que se consideran claves en el futuro inmediato, la prospectiva tecnológica ofrece "información estratégica difícil de adquirir que sirve para la toma de decisiones, y funciona como un instrumento de movilización socioeconómica para fomentar la sensibilización y crear un consenso en torno a formas prometedoras de explotar las oportunidades y reducir los riesgos de la evolución científica y tecnológica" (Morato, Rodríguez, Miles, Keenan, Clar, Svanfeldt, 2002, 5).

Por ello, es necesario analizar las características básicas de la prospectiva tecnológica y su relación con las políticas de I+D e innovación, deteniéndonos específicamente en el contexto europeo y español. Después de esta introducción, el artículo consta de cinco puntos, que siguen la siguiente secuencia lógica: analizar el margen de actuación ante el desarrollo e implantación de trayectorias tecnológicas, describir la metodología prospectiva, analizar el papel estratégico que juega en nuestro sistema de I+D e innovación, presentar algunos de los principales resultados de dichas investigaciones en España y la Unión Europea, y finalmente concluir señalando sus limitaciones y posibilidades. Específicamente, en el punto número dos abordaremos los modelos de desarrollo socio-técnicos, y los impactos que se derivan de las decisiones que tomamos en el presente sobre el futuro inmediato. En el punto número tres, describimos las características básicas de los estudios prospectivos, las metodologías y los objetos de análisis. En el punto número cuatro, analizaremos la dimensión estratégica de la prospectiva, al aportar información cualificada que permite anticiparnos a los riesgos y oportunidades derivados de la innovación tecnológica. En el punto número cinco, presentaremos las principales investigaciones prospectivas que se han realizado en España y en la Unión Europea. Y, finalmente, en las conclusiones, señalaremos algunas cuestiones clave que deben tomar en consideración los estudios de prospectiva en el ámbito de las políticas de I+D e innovación.

\section{TeCnología, prospectiva y políticas de I+D E INNOVACIÓN: ¿ES POSIBLE SUPERAR EL DILEMA de Collingridge?}

La política de I+D e innovación en una sociedad tecnológica avanzada demanda información cualificada sobre el futuro, para poder elaborar una planificación a corto, medio y largo plazo, por tres razones: en primer lugar, es 
necesario anticipar las características del modelo tecnológico en un horizonte a diez o quince años. Por ejemplo, establecer un modelo de producción, distribución y consumo de energía en un país supone planificar, diseñar, construir y poner en funcionamiento un conjunto de instalaciones de producción y redes de distribución en función de las previsiones realizadas. En segundo lugar, los errores en la previsión del futuro generan graves problemas de forma inmediata: "los fracasos a la hora de anticipar cambios sutiles o acumulativos a largo plazo tienen consecuencias repentinas a corto plazo" (Abt, 2003, 88). En tercer lugar, es necesario establecer un proceso de reevaluación continua de las previsiones y los resultados, para ajustarse a los cambios que se producen en el entorno: "el objetivo se convierte así en prepararse para tomar las decisiones más pertinentes en el momento oportuno, más que en adivinar con exactitud el futuro" (Schwartz, 2003, 37).

Planificar significa pronosticar, evaluar, elegir prioridades, y optar en función de los recursos disponibles. Las discusiones sobre los límites de nuestras metodologías para anticipar el futuro no pueden hacernos olvidar que estamos inmersos en un sistema científico tecnológico en el que estamos financiando las innovaciones que estarán en el mercado dentro de diez años, y que la capacidad para hacer frente a riesgos presentes y futuros dependen de nuestra planificación estratégica, y de cómo actuamos ahora para afrontar un riesgo emergente en los próximos años. No es extraño, por ello, que los estudios de futuro se desarrollaran en los años cincuenta en el entorno de la Guerra Fría, y en el ámbito militar, en un contexto de innovación tecnológica (la energía nuclear y la carrera espacial) que podía afectar al equilibrio de poder existente. En la actualidad, la mayor parte de los países miembros de la Unión Europea, y muchos países de otras regiones del mundo, realizan sistemáticamente estudios prospectivos, con la finalidad de aportar información cualificada para la toma de decisiones en el ámbito de la política científico-tecnológica.

En sociedades democráticas, el análisis de los riesgos tiene, además, otra consecuencia directa: los ciudadanos quieren tener más información, y una mayor participación, en la evolución e implantación de tecnologías que les afectan en su vida cotidiana. El análisis del futuro no puede dejar de lado las consecuencias sociales de los desarrollos previstos, y el papel clave que juega cada ciudadano como consumidor (en el caso de productos) o como decisor político (en el caso de tecnologías que afectan a la definición de nuestra propia identidad como seres humanos). De ahí que las metodologías de los estudios de futuro incluyan cada vez más el análisis de los impactos sociales, y permitan aumentar la información disponible para la toma de decisiones.

Esto es más relevante en la medida en que, como muestra el denominado dilema de Collingridge (Collingridge, 1980), es muy difícil tomar decisiones para orientar o configurar una tecnología en los momentos iniciales de su desarrollo, ya que sabemos poco acerca de sus costes, oportunidades, riesgos y efectos positivos y negativos. Sin embargo, cuando se ha desarrollado e implantado, y tenemos suficiente conocimiento sobre ella, es muy difícil introducir modificaciones en su trayectoria. Una posible forma de superar este dilema es aportar, mediante la metodología prospectiva, información adecuada antes de que las trayectorias tecnológicas sean irreversibles. Por ello, los estudios prospectivos se han convertido en una herramienta estratégica, tanto para la competitividad interna de los países, cuanto para el sistema democrático mismo, en la medida en que las opciones tecnológicas que tomamos, o que dejamos de tomar, configuran de manera a veces definitiva nuestro modelo de vida.

\section{3. ¿UN NUEVO CAMPO DE INVESTIGACIÓN CIENTÍFICA? La INSTITUCIONALIZACIÓN DE LA PROSPECTIVA Y LOS FUTURES STUDIES}

Los estudios sobre el futuro, y lo que denominamos "prospectiva" (definida por la Real Academia de la Lengua como "conjunto de análisis y estudios realizados con el fin de explorar o predecir el futuro de alguna materia"), se han desarrollado hasta el punto de configurar un área temática propia (Bass, 1998). La consolidación de una comunidad científica está directamente relacionada con la edición de revistas especializadas (Futures, Technological Forecasting and Social Change), la creación de organizaciones de profesionales que comparten resultados y debaten enfoques (World Future Society, World Future Studies Federation), y la institucionalización de estos enfoques en las universidades y los institutos de investigación públicos y privados. Desde los años 1960, se han llevado a cabo una gran diversidad de estudios prospectivos, vinculados a tecnologías concretas, a la posición competitivas de empresas, regiones

ARBOR CLXXXV 738 julio-agosto [2009] 825-836 ISSN: 0210-1963

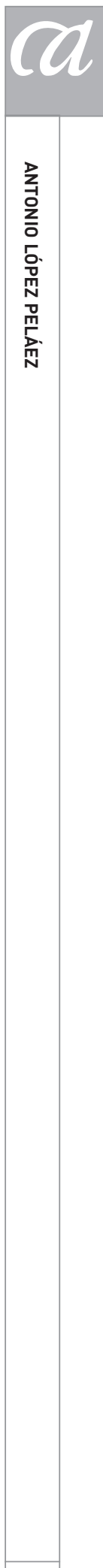

827 
y paises, y a los impactos sociales de las innovaciones tecnológicas, configurando un amplio espectro de investigaciones relevantes (Keenan, 2003). Además, progresivamente el ámbito de los estudios prospectivos se ha ido ampliando, más allá de la prospectiva tecnológica estrictamente considerada, tomando en consideración cuestiones relevantes para la política de I+D e innovación, como el mercado de trabajo, la formación de recursos humanos, o la tendencias de evolución de sectores clave como el turismo, los servicios sociales y el estado del bienestar, o el sistema sanitario (Häder y Häder, 2000).

El punto de partida de dichos estudios es la consideración del futuro como un producto de procesos sociales, que no está predeterminado necesariamente de antemano (Ringland, Todd, Schwartz, 1998): existen diversos futuros posibles, con diversos grados de probabilidad, y que conllevan consigo diferentes consecuencias sobre la sociedad. Precisamente por ello, se trata de presentar críticamente los diferentes escenarios alternativos, para aumentar la información rigurosa sobre la toma de decisiones, y contribuir así a la construcción social del futuro. Las investigaciones sobre el futuro aumentan la información disponible para afrontar las posibilidades y riesgos que conllevan nuestras elecciones. Los resultados de la investigación prospectiva introducen, en los procesos de toma de decisiones, información relevante relativa a los previsibles impactos y consecuencias que se derivan de cada una de las opciones posibles que se pueden tomar (Gries, 2000, 24).

Como en cualquier otro enfoque, o disciplina científica, una clave esencial es el método. Los Future Studies se consolidan no sólo por su objeto de estudio (analizar el futuro), sino por la metodología diseñada para hacerlo, en la que juegan un papel fundamental los expertos en desarrollo tecnológico (que trabajan con un horizonte a corto, medio y largo plazo, en función de los objetivos que pretenden alcanzar). Tras la Segunda Guerra Mundial, la tecnología aparece como el factor competitivo básico para obtener el poder militar y el poder económico. En el contexto de la Guerra Fría, los primeros estudios prospectivos en los Estados Unidos de América se desarrollan en el ámbito militar, en el que la innovación tecnológica y el diseño de planes de respuesta a una acción del enemigo implican una perspectiva temporal estratégica, en torno a la cual hay que establecer planes de acción. Sin embargo, los estudios prospectivos rápidamente ampliaron su ámbito de aplicación, abordando cuestiones vinculadas con la evolución económica (Jouvenel, 1967), el cambio social, la innovación tecnológica, la gestión de catástrofes previsibles (Gabor, 1963), o los límites del crecimiento (Meadows, 1972).

La misma RAND Corporation, en la que se desarrolló el método Delphi (Landeta, 1999), y vinculada en sus orígenes al campo militar, desde 1948 "paso de pronosticar sistemas alternativos de armas a tener una perspectiva más sociológica, centrada en el bienestar social y la política" (Bass, $1998,23)$. En nuestras sociedades tecnológicas avanzadas, o sociedades del conocimiento, las investigaciones prospectivas se aplican, cada vez más, a un mayor número de ámbitos: desde el análisis de la evolución de tecnologías como la robótica (López y Kyriakou, 2008), hasta la prospectiva ocupacional: "prácticamente todos los gobiernos de los paises avanzados y las agencias internacionales relacionadas con el mundo del trabajo realizan pronósticos sobre la fuerza laboral del futuro y la distribución del empleo entre los sectores industriales y las ocupaciones" (Freeman, 2003, 177).

Podemos destacar dos características básicas de los estudios prospectivos: en primer lugar, su marcado carácter interdisciplinar. En segundo lugar, la ampliación progresiva de su ámbito de análisis: desde la evolución de las trayectorias tecnológicas, hasta el análisis de macro-tendencias económicas, políticas o sociales. Si nos limitamos a considerar la importancia de las investigaciones prospectivas en el sistema de I+D e innovación de nuestras economías avanzadas, es posible diferenciar tres grandes líneas de análisis prospectivo. En primer lugar, los estudios sobre prospectiva tecnológica, vinculados a la financiación de tecnologías clave, y que suelen establecer la posición competitiva de una empresa, región, o país, en relación a otros competidores [los sistemáticos estudios Delphi japoneses podrian considerarse un modelo (NISTEP, 2005)]. En segundo lugar, la investigación sobre los procesos de innovación tecnológica y los cambios que se derivan de los desarrollos previstos en tecnologías clave, como la ingeniería genética, la robótica o las tecnologías de la información y la comunicación. Finalmente, en tercer lugar la investigación sobre la interacción entre la innovación tecnológica y las demandas sociales, analizando los riesgos, oportunidades e impactos que se derivan de las trayectorias tecnológicas probables, desde una perspectiva centrada en la recepción de dichas tecnologías y los problemas de implantación prácticos. 
De acuerdo con su objeto de estudio, las investigaciones prospectivas utilizan metodologías de investigación cuantitativas y cualitativas, como las encuestas o las entrevistas a expertos, y también han generado nuevos métodos de investigación, como el método Delphi. Podemos agrupar las diferentes metodologías en tres grandes áreas (Keenan, Butter, Sainz de la Fuente, Popper, 2006). En primer lugar, aquellas que se han desarrollado especificamente para proporcionar información sobre el futuro, y que toman en consideración las previsiones de los expertos como protagonistas de la propia evolución tecnológica: la elaboración de escenarios ${ }^{1}$, el método Delphi ${ }^{2}$ la elaboración de mapas de evolución tecnológica ${ }^{3}$, o el MIC (matriz de escenarios cruzados) $)^{4}$. En segundo lugar, los métodos basados en el análisis de datos, y que permiten realizar proyecciones y simulaciones sobre la evolución previsible de los acontecimientos. En tercer lugar, otras aproximaciones vinculadas con el análisis de las fuentes documentales, las entrevistas o los workshops, que se utilizan tanto en la prospectiva como en otro tipo de investigaciones científicas.

\section{Construir el futuro en un entorno cambiante: EL VALOR ESTRATÉGICO DE LA PROSPECTIVA}

Las sociedades basadas en la tecnociencia se caracterizan por la anticipación del futuro, que se convierte en el referente que determina la acción, desarrollando estrategias para reducir la incertidumbre y asegurar la viabilidad no solo económica, sino social, a corto, medio y largo plazo. "Las sociedades modernas han entronizado el cambio y no la estabilidad, la innovación y no la repetición como mecanismos de adaptación (...). El futuro, no el pasado, controla el presente. $Y$ por ello, la menor validez de los modelos normativos para explicar la conducta y, por el contrario, la mayor importancia de las especificas definiciones de la situación por los actores. La indagación del futuro es ya parte primordial de la socialización cotidiana que requiere conocer lo que va a ocurrir para posicionarse frente a ello" (Lamo, González y Torres, 1995, 41).

En este sentido, uno de los objetivos de los estudios prospectivos es prever los "eventos" que se materializarán en períodos temporales concretos, presentar las "tendencias" de desarrollo probables, y elaborar "escenarios" de futuro. Estos escenarios no son simplemente posibles, sino alta- mente probables (Georgantzas y Azcar, 1995). Aunque hay desarrollos tecnológicos que no pueden ser anticipados, gran parte de las innovaciones tecnológicas que se implantarán en los próximos años están actualmente en período de desarrollo, y sobre ellas se pueden elaborar proyecciones tecnológicas altamente probables. Las innovaciones tecnológicas pueden producir catástrofes o utopias, en función de cómo nos preparemos para ellas. $Y$ hay que tener en cuenta que la velocidad de los avances tecnológicos lleva a que las previsiones sobre implantación de tecnologías que alterarán nuestra forma de vida de manera radical se sitúan en un horizonte temporal a medio plazo (20 años), y se desarrollarán por lo tanto durante la vida de más de la mitad de la población actual (Kaplan, 1994).

Ante este horizonte de cambios, la función principal de las predicciones consiste en aportar información, para poder realizar un análisis racional de las tendencias de futuro, y así evitar depender completamente del azar. Este análisis debe tener en cuenta las consecuencias sociales positivas y negativas, para mitigarlas o facilitarlas cuando se presenten. Por ello, en base a los factores sociales, políticos y morales que guian nuestras elecciones tecnológicas, se pueden formular escenarios que vayan desde la perspectiva optimista hasta la pesimista, y que nos permitan establecer un debate sobre el futuro de nuestras sociedades. Como señala Warren Wagar al establecer tres escenarios secuenciales que prevé se implantarán progresivamente en las sociedades del siglo XXI (un escenario tecnoliberal, un escenario radical socialista y un escenario contracultural y utópico-ideológico), las respuestas sociales y políticas al desarrollo científico-tecnológico son consecuencia del agotamiento de los sistemas actuales de gestión de la economía y la tecnología (precisamente por su incapacidad para gestionar los retos de las nuevas tecnologías y los nuevos riesgos). Pero, a la vez, no prefiguran un modelo único de respuesta, y tienen que ver con los valores y criterios socialmente vigentes (Wagar, 1991).

La diferencia fundamental entre la previsión en un sistema físico y un sistema social estriba en la naturaleza misma de lo social. Los actores sociales intervienen en el desarrollo de la sociedad, y por tanto cualquier previsión sobre el futuro será intrínsecamente imperfecta en relación a las previsiones que se pueden realizar en los sistemas físicos. Ahora bien, el objetivo final de las previsiones en los sistemas sociales no es tanto determinar mecánicamente

ARBOR CLXXXV 738 julio-agosto [2009] 825-836 ISSN: 0210-1963

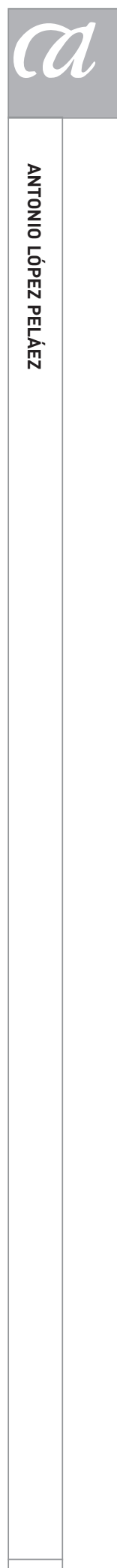


el futuro. Más bien, se trata de aportar información para intervenir en la construcción social de dicho futuro, en función de los impactos previsibles y del modelo social que se considere deseable. En este sentido, la investigación prospectiva no debe aportar solamente previsiones generales, sino también debe identificar fines "deseables y realizables". Dado que la especie humana configura su futuro, se puede, en primer lugar, predecir un futuro humano altamente probable; $y$, en segundo lugar, se puede evitar un futuro indeseable. Al constituir los desarrollos tecnológicos una de las principales vías por las cuales la humanidad afecta/configura su futuro, es conveniente anticipar dichos desarrollos, y establecer estrategias para la construcción del futuro. La elaboración de los escenarios, en base a las previsiones formuladas por los expertos, puede ordenarse en torno a los dos extremos posibles: la utopia (el escenario más deseable dentro de los diversos futuros probables) y la distopía (es escenario menos deseable dentro de los diversos futuros probables).

Las previsiones sobre el futuro de nuestras sociedades parte, pues, de un principio básico: las personas y las organizaciones son capaces de usar la ciencia y la tecnología de forma provechosa, ventajosa, y que beneficie también a las próximas generaciones. Este principio implica que esta posibilidad siempre va acompañada de su contrario: las capacidades tecnológicas pueden ser usadas de forma no provechosa. La capacidad de influir en el futuro, como fundamento de los análisis prospectivos, va unida al papel fundamental que juegan la ciencia y la tecnología en la construcción de dicho futuro. De ahí la relevancia de las previsiones de los expertos, que nos muestran los avances tecnológicos que se materializarán en los próximos años. Los resultados de los análisis prospectivos deben ir dirigidos, por lo tanto, a un público compuesto por las personas responsables en la planificación tecnológica, económica y social. Pero también deben ir dirigidos a la ciudadanía, para que analice las potenciales oportunidades y riesgos. En este sentido, la evaluación de tecnologías se abre a la opinión pública, contribuyendo al debate sobre la construcción democrática de la sociedad tecnológica del mañana.

En el ámbito de la investigación sobre el futuro, debe diferenciarse entre el pronóstico, y la predicción (Coates, Mahaffie, Hines, 1997, 4): el pronóstico puede definirse como "un simple o complejo análisis de las cualidades y probabilidades de un evento futuro o tendencia". Y la predicción puede definirse como "una afirmación específica y usualmente cuantitativa sobre un resultado futuro". A partir de las previsiones y los pronósticos, pueden elaborarse escenarios: necesitamos formular asunciones acerca del futuro para ordenarlo, prepararlo, y prevenir eventos indeseables que puedan ocurrir. Los escenarios se basan en estas "asunciones" acerca del futuro. Es decir, sobre estas afirmaciones altamente probables acerca del futuro, que forman una estructura o sistema, se pueden establecer consecuencias acerca del futuro, y formular el modelo de sociedad que se deriva de ellas. En los escenarios, se trata de buscar una convergencia de evidencias y eventos altamente probables, puesto que no hay un determinismo tecnológico que implique desarrollos inevitables. En este sentido, junto a los descubrimientos científicos y las aplicaciones tecnológicas deben tenerse en cuenta los factores contextuales: sociales, económicos, políticos, ambientales, militares, etc., que conducen, forman e influyen en la emergencia de las sociedades tecnológicas avanzadas.

La prospectiva ofrece, por lo tanto, un conjunto de escenarios posibles, que se derivan de la interacción entre los determinismos del pasado y la confrontación de los proyectos de los actores sociales que intervienen en el desenvolvimiento del futuro. No son, por lo tanto, escenarios elegidos al azar, y no son improbables. Son hipótesis coherentes sobre el futuro posible, a las que se puede asignar un determinado grado de probabilidad (Godet, 1991). En el ámbito concreto de la prospectiva tecnológica, la previsión tiene como objetivo establecer un mapa de las tecnologías emergentes, para orientar el desarrollo científico de una empresa o país en la dirección adecuada. Por ello, puede definirse la prospectiva tecnológica como un conjunto de "tentativas sistemáticas para observar a largo plazo el futuro de la ciencia, la tecnología, la economía y la sociedad con el propósito de identificar las tecnologías emergentes que probablemente produzcan los mayores beneficios económicos y/o sociales" (OPTI, 1999, 10). En este sentido, un amplio elenco de países, como EE.UU., Alemania, Austria, Reino Unido, Australia, Francia, Japón y España, utilizan la prospectiva como una herramienta clave en el diseño de sus políticas de I+D e innovación. El análisis de las investigaciones prospectivas desarrolladas en estos países a lo largo de los últimos 30 años nos permite establecer algunas conclusiones: 
- En primer lugar, la prospectiva es una actividad de carácter estratégico: "aplicada al campo de la ciencia y la tecnología, la prospectiva permite identificar tendencias de evolución del conocimiento, situando siempre estas tendencias en contextos de acción concretos. Por ello, constituye una herramienta básica para la definición de estrategias y políticas relacionadas con la I+D, y así se está empleando desde hace décadas en la mayoría de los países industrializados" (ENCYTE, 2008, 10). Permite explotar los conocimientos sobre el futuro, para hacer posible un desarrollo científico-tecnológico que permita aumentar el bienestar de la sociedad.

- En segundo lugar, la prospectiva permite elaborar una base de información racional sobre las tendencias de desarrollo científico-tecnológico. Supone, por lo tanto, una transformación radical en el análisis y planteamiento de las acciones de los actores sociales, al introducir un conjunto de informaciones sobre los desarrollos tecnológicos futuros, que pueden orientar sistemáticamente la acción, y pueden ayudar a establecer prioridades y a lograr un mayor nivel de previsión y anticipación de los riesgos. Los análisis prospectivos permiten establecer vías concretas de actuación, en base a la información sobre las tecnologías emergentes y las áreas científicas más relevantes. Al integrar las opiniones de los expertos, estableciendo rigurosamente previsiones de eventos y tendencias, y elaborando escenarios posibles, permite crear una "base de información racional que puede, y debe, ser utilizada para recomendar acciones a los responsables de $I+D$, para decidir prioridades de financiación o vías de desarrollo, que cuentan así con un respaldo objetivo a las decisiones a tomar en el desempeño de sus funciones" (OPTI, 1999, 10).

- En tercer lugar, en el ámbito de los impactos sociales de los desarrollos científico-tecnológicos, la prospectiva nos ofrece información relevante para establecer secuencias de impactos posibles, con un determinado nivel de seguridad en la previsión. Permite anticipa los impactos y transformaciones sociales que se derivarán del desarrollo científico y tecnológico en los próximos años. Es decir, trata de "(..) analizar las tendencias de cambio más claramente identificables en nuestra sociedad y formular los escenarios alternativos a los que dichas tendencias pueden conducirnos" (Castilla, 2000, 47). A partir de esta información, es posible anticipar estrategias que minimicen los costes sociales generados por la implantación y desarrollo de las nuevas tecnologías. $Y$, a la vez, permite plantear desde una nueva perspectiva (los escenarios futuros posibles) el debate sobre la construcción de la sociedad tecnológica, y sobre los límites que conlleva la forma actual de diseñar y gestionar el desarrollo tecnológico. Con ello, ayuda a mostrar las limitaciones intrínsecas de los actuales modelos de desarrollo económico y de organización social, enfrentándolos a las transformaciones y contradicciones que generarán en los próximos años los avances tecnológicos.

En función de estas tres características, la investigación sobre el futuro ayuda a establecer un debate sobre los valores que intervienen en la construcción social del futuro, aportando un nuevo punto de vista: un conjunto de previsiones sobre los avances tecnológicos y sus impactos en un horizonte temporal a corto, medio y largo plazo. Desde esta perspectiva sobre el futuro, y sus consecuencias probables, es posible analizar los sistemas de organización económica y social. Y las contradicciones o consecuencias que se derivarán de los impactos sociales que genera la aplicación de las nuevas tecnologías desde los valores que articulan dichos sistemas de organización económica y social.

\section{La prospectiva en España y en la Unión EUROPEA}

La investigación sobre el futuro se ha convertido, en las sociedades tecnológicas avanzadas, en una cuestión estratégica, y una consecuencia directa es el aumento exponencial de los estudios prospectivos financiados por diversas instituciones en todas las áreas geográficas. Solamente en el período 2004-2006, se han clasificado más de 1.400 investigaciones en este campo en todo el mundo. Un análisis detallado de las mismas nos permite formular las siguientes conclusiones (Keenan, Butter, Sainz de la Fuente, Popper, 2006, 3-6):

- En primer lugar, en el ámbito geográfico de la Unión Europea se han desarrollado más de un tercio del total de investigaciones analizadas, lo que indica el papel relevante de la prospectiva para los países que componen la Unión Europea, y la importancia estratégica que tiene crear una red europea que permita aprovechar las potencialidades de las investigaciones que se realizan, y trabajar con una mentalidad europea, más allá de los intereses nacionales o regionales. Las otras dos grandes

ARBOR CLXXXV 738 julio-agosto [2009] 825-836 ISSN: 0210-1963

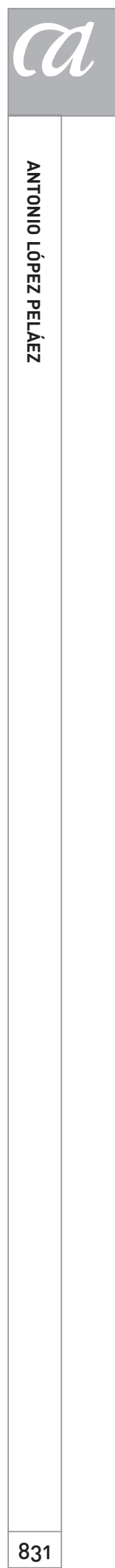


áreas geográficas relevantes en el campo de la prospectiva son Asia (con Japón como país líder) y América del Norte, con un crecimiento importante de los estudios prospectivos en América del Sur.

- En segundo lugar, en todas las áreas geográficas el principal financiador y/o cliente son los gobiernos, regionales, nacionales o supranacionales. Sin embargo, en los Estados Unidos de América y en Australia, hay un número relativamente alto de investigaciones financiadas por empresas privadas.

- Los métodos utilizados son muy variados, destacando el predominio del método Delphi en Asia (especialmente en el caso de Japón), la elaboración de escenarios (muy utilizado en la Unión Europea), y el análisis de tecnologías clave (key technologies) y mapas de evolución tecnológica (technology road mapping -TRM-) (métodos más frecuentemente utilizados en los Estados Unidos de América). Sin embargo, hay que señalar que, dado el enfoque multidisciplinar propio de la disciplina, las investigaciones prospectivas suelen utilizar múltiples métodos en función de su objeto de estudio.

-El ámbito geográfico de los estudios es, en su mayoría, de ámbito nacional. Sin embargo, en la Unión Europea se realizan también un destacable número de investigaciones de ámbito regional (Keenan, Butter, Sainz de la Fuente, Popper, 2006, 9).

-El mayor porcentaje de estudios se ha realizado sobre las siguientes áreas: manufactura, transporte, agricultura y alimentación, salud, tecnologías de la información y la comunicación, energía, tecnología medioambiental, e investigaciones sobre el área denominada ciencias sociales y humanidades (servicios sociales, sistemas públicos y privados sanitarios, turismo, mercado de trabajo, formación y recursos humanos, etc.) (Ziglio, 1996; Häder y Häder, 2000; Keenan, Butter, Sainz de la Fuente, Popper, 2006).

- Los principales resultados son los siguientes: análisis de tendencias, elaboración de escenarios, mapas de evolución de tecnologías clave, prioridades de investigación, y recomendaciones para guiar los programas de inversión de I+D e innovación públicos y privados.

Disponer de información cualificada para la toma de decisiones, que permitan priorizar las inversiones, y proporcionar una visión adecuada de la evolución de la ciencia y la tecnologia, desde la investigación básica hasta los impactos sociales, se ha convertido en cuestión estratégica (NISTEP, 2006, 2). Así lo reconoce la propia Unión Europea, al crear instituciones de investigación que proporcionan información válida para la toma de decisiones, como el Instituto de Prospectiva Tecnológica (IPTS), financiar mediante el programa COST (Action A22) la European Network for Foresight Methodology, integrada por más de 150 miembros de 23 países (Fuller, Smedt, Rothman, 2006, 3-4), crear la denominada European Foresight Knowledge Sharing Platforma (KSP), y organizar los seminarios FTA (Future-Oriented Technology Analysis) (convocatoria bianual que se celebra en la sede del IPTS en Sevilla, desde el año 2004) (Scapolo, Porter, Rader, 2008). Desde el punto de vista de la política científica de la Unión Europea, las áreas clave sobre las que deben realizarse análisis prospectivos son las siguientes: Biotecnología, Sociedad de la Información, Transporte, Energía, Desarrollo sostenible e Industria. Entre los proyectos de investigación actualmente en desarrollo, podemos destacar el proyecto Europolis (Scenarios for the Evaluation of the European Science and Technology Policy), en el que el análisis prospectivo tiene como objetivo analizar la evolución previsible de la ciencia y la tecnología en Europa, y debatir nuevas orientaciones e iniciativas que favorezcan una adecuada política de I+D e innovación.

España, dentro de la Unión Europea, se caracteriza por un progresivo incremento de las investigaciones en el ámbito de la prospectiva tecnológica, tanto a nivel nacional como regional, incluyendo las líneas de investigación de universidades y empresas. Sin embargo, aún no se ha alcanzado el nivel de otros países de la Unión Europea, especialmente en el ámbito de la política científica. De hecho, podemos señalar que la incorporación de la prospectiva, y el desarrollo de políticas científico-tecnológicas basadas en la evidencia, constituye todavía hoy un reto para nuestras agencias de la política científica. Podemos sintetizar brevemente el estado de la investigación prospectiva en España diferenciando tres ámbitos:

- En primer lugar, en el ámbito institucional, hay que resaltar la labor realizada por Fundesco, lamentablemente desaparecida, en torno al grupo de trabajo liderado por Adolfo Castilla. En la década de 1970 se creo el Instituto Nacional de Prospectiva, en el que desempeño un papel revelante el profesor Emilio Fontela. Posteriormente, el Observatorio de Prospectiva Tecnológica Industrial (OPTI), dependiente del Ministerio de Industria, ha desarrollado estudios prospectivos sistemáticos sobre tecnologías clave desde el año 1999. A partir del año 2007, se establece 
una Estrategia Nacional de Ciencia y Tecnología (ENCYT). Se trata de un documento de posición diseñado por el Gobierno de España y acordado por unanimidad en la III Conferencia de Presidentes Autonómicos celebrada el 11 de enero de 2007, en el que se recogen los grandes principios y objetivos generales que han de regir las políticas de ciencia y tecnología, tanto nacionales como regionales, en el horizonte temporal 2007-2015. Sus objetivos son los siguientes: situar a España en la vanguardia del conocimiento, promover un tejido empresarial ampliamente competitivo, integrar los sistemas regionales en el Sistema de ciencia y tecnología, potenciar la dimensión internacional del Sistema de ciencia y tecnología, disponer de un entorno favorable a la I+D+i, y disponer de las condiciones adecuadas para la difusión de la ciencia y la tecnología. Para poder abordar estos objetivos, en función de las tendencias de evolución futura, la Fundación para la Ciencia y la Tecnología (FECYT), a través del Observatorio permanente del sistema español de ciencia-tecnología-sociedad, ha publicado en 2008 el informe Estrategia Nacional de Ciencia y Tecnología. Ejercicio de prospectiva a 2020. Las áreas tecnológicas analizadas son las siguientes: Agroalimentación y Pesca, Ciencias de la Salud y Biomedicina, Energía, Humanidades y Ciencias Sociales, Ordenación del Territorio y Recursos Turísticos, Química, Materiales, Recursos Naturales y Medio Ambiente, Seguridad y Defensa, Tecnologías de la Información y las Comunicaciones, Tecnologías de Diseño y Producción Industrial, Transporte, Matemáticas, y Física y Ciencias del Espacio. En cada una de estas áreas, las dimensiones analizadas son las siguientes: Tendencias identificadas, Datos generales de la encuesta, Líneas prioritarias, y Ciclo de desarrollo. La investigación prospectiva, por lo tanto, es considerada en España, como en el resto de la Unión Europea, una herramienta estratégica para orientar la acción política en el ámbito de la ciencia y la tecnología. Así también se percibe desde los diferentes gobiernos autonómicos, algunos de los cuales han desarrollado programas específicos de investigación prospectiva en los últimos años (Morato, Rodríguez, Miles, Keenan, Clar, Svanfeldt, 2002).

- En segundo lugar, las líneas de investigación prospectivas que se desarrollan en universidades y empresas privadas. En el ámbito empresarial, podemos destacar, entre otras, las investigaciones del Grupo Telefónica. En el ámbito académico, es necesario hacer referencia al proyecto de investigación "Tendencias sociales de nuestro tiempo" (dirigido por el catedrático de Sociología de la UNED profesor José Félix Tezanos), desde 1995 hasta la actualidad, y radicado institucionalmente en la Fundación Sistema. Se trata del proyecto de investigación prospectiva más relevante realizado en España en el ámbito de la robótica, las biotecnologías y la ingeniería genética, y las tecnologías de la información y la comunicación (Tezanos, 2007). Pero también ha abordado otras áreas, como las tendencias políticas, económicas y sociales (Tezanos y Villalón, 2002, 215-220). Combinan encuestas (se han realizado hasta la fecha 13 encuestas generales sobre tendencias sociales), estudios delphi (se han llevado a cabo 31 estudios delphi, sobre diversos temas, y tres tandas de estudios delphi sobre tendencias científico-tecnológicas -en 1996, 2002-2003 y 2005-), estudios de caso, y sus resultados se han publicado tanto en editoriales y revistas españolas, como en revistas internacionales prestigiosas en el área (Futures, Technological Forecasting and Social Change, The IPTS Report, Social Epistemology).

- En tercer lugar, hay que resaltar las jornadas, encuentros y foros académicos relacionados con la prospectiva, y la publicación de obras de referencia en este campo. Ya desde el inicio de la década de 1970, la investigación sobre el futuro tuvo una destacable acogida en España, gracias al número monográfico sobre "El futuro: previsión y política", coordinado por el profesor Salustiano del Campo, y publicado en 1970 por la Revista Española de la Opinión Pública. En este monográfico se presentaban, junto con la descripción de las investigaciones realizadas por la RAND Corporation, dos artículos sobre la metodología Delphi escritos por sus creadores (Helmer, 1970). Posteriormente, podemos destacar, entre otros, algunos textos de referencia sobre la metodología de los estudios prospectivos (Bass, 1999; Landeta, 1999), la primera conferencia española de prospectiva organizada por la Universidad de Alicante y dirigida por el profesor Enric Bas en el año 2002, y los Foros de Tendencias Sociales organizados por el Grupo de Estudios de Tendencias Sociales (GETS) en la UNED desde 1995 hasta la actualidad, y editados por la Editorial Sistema (Tezanos, 2008).

\section{Conclusiones}

En nuestras sociedades avanzadas, como hemos podido analizar, las políticas de I+D e innovación se han convertido

ARBOR CLXXXV 738 julio-agosto [2009] 825-836 ISSN: 0210-1963

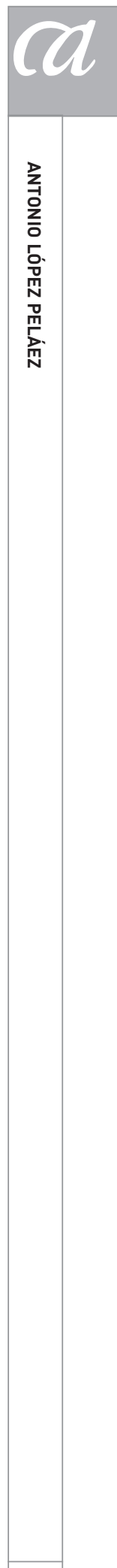

833 
en una cuestión estratégica. La toma de decisiones en este ámbito tiene que hacer frente a tres grandes retos. En primer lugar, establecer los objetivos deseables a alcanzar, en función de los recursos de la empresa, del país o de la región geográfica de la que se trate. En segundo lugar, hacer frente a los riesgos que se derivan tanto de las consecuencias esperadas o inesperadas de nuestras decisiones, como de la evolución del resto de actores con los que competimos. En tercer lugar, favorecer el desarrollo de las tecnologías clave en los próximos años. Todo ello en un entorno de incertidumbre, en el que deben reevaluarse las previsiones y los resultados cada cierto tiempo, y en el que los recursos financieros son limitados. Es evidente que las decisiones que tomamos configuran nuestro futuro posible, y dado el elevado coste que podemos sufrir en todos los órdenes, no podemos adoptar una posición pasiva. Necesitamos abordar las tendencias de cambio, necesitamos trabajar con las previsiones de los expertos. En definitiva, la consolidación de la Prospectiva y los denominados Future Studies se produce en una sociedad volcada hacia el futuro, y que demanda información cualificada para la toma de decisiones.

Sin embargo, es necesario también considerar las limitaciones de los análisis prospectivos. Podemos analizar, brevemente, las siguientes: En primer lugar, la necesidad de contar con expertos cualificados en los temas que se investigan. Tanto en el método Delphi como en la elaboración de escenarios, o en los mapas (u hojas de ruta) de evolución tecnológica (TRM), el concurso de expertos bien seleccionados constituye un requisito indispensable, y puede suponer un severo problema en función de la densidad de una comunidad cientifica determinada (por ejemplo, en el estudio Delphi realizado por el Ministére de l'Enseignement Supérieur et de la Recherche de Francia, en 6 de los 21 eventos propuestos en el área de la Robótica ninguno de los expertos seleccionados se consideró altamente cualificado para responder (MESR, 1995; López Peláez, 2003:
208-219)). En segundo lugar, hay que tener en cuenta que lo que los actores piensan sobre el futuro no es la única variable que interviene en su materialización efectiva, aunque en el ámbito de las trayectorias tecnológicas sea más fácil realizar proyecciones basadas en el juicio experto, que en otros ámbitos de las ciencias sociales. En tercer lugar, no hay que olvidar que las previsiones se realizan, a menudo, para aportar información y que no se cumplan, en la medida de lo posible, los impactos negativos previstos. En cuarto lugar, las trayectorias tecnológicas, al desarrollarse, tienen consecuencias que van más allá de la simple incorporación de un aparato o una tecnología: cambian el medio social, y por lo tanto las previsiones tienen que reformularse con rapidez. De ahí que sea necesario realizar estudios prospectivos no de forma aislada, sino dentro de un plan sistemático de investigación, ya que los resultados obtenidos pueden volverse rápidamente obsoletos.

Los principales retos de las investigaciones prospectivas están relacionados con las siguientes cuestiones: En primer lugar, el desarrollo de metodologías cada vez más eficaces, en la línea de lo que se denomina Future-oriented technology analyses (FTA). En segundo lugar, una mayor coordinación entre las instituciones locales, regionales, nacionales y supranacionales, que permitan compartir esfuerzos y resultados. En tercer lugar, una mejor diseminación de los resultados, especialmente entre las empresas que pueden aprovechar las tendencias previstas para reforzar su posición competitiva. En la medida en que el cambio tecnológico se acelera, y sus impactos sociales son mayores, la prospectiva tecnológica se convierte en una herramienta indispensable para configurar el futuro posible. En este sentido, en los próximos años, el objetivo básico de la Unión Europea en este ámbito, convertirse en la mayor economía del conocimiento y de la innovación, va a reclamar más y mejores estudios prospectivos para orientar las políticas de I+D e innovación.

\section{NOTAS}

1 "Un escenario es un futurible, un futuro posible de entre varios alternativos, que describe una situación hipotética futura (...). El método de escenarios

Recibido: 10 de septiembre de 2008 Aceptado: 25 de enero de 2009 es anticipatorio, en tanto en cuanto ofrece un mapa cognitivo del futuro que contiene una serie de alternativas de futuro (escenarios) posibles, cada una de las cuales tiene asignada una probabilidad, objetiva o subjetiva, de ocurrencia" (Bass, 1999: 110-111).

2 El método Delphi es un método de investigación dirigido a la obtención de una previsión consensuada y fide- 
digna por parte de un grupo de expertos, a través de varias rondas de un cuestionario (dos o tres, aunque normalmente se reduce a dos rondas) (Landeta, 1999: 101-102). Su objetivo es investigar, de manera rigurosa, el conjunto de conocimientos, experiencias y habilidades de los expertos en una determinada cuestión, de cara a aumentar la información crítica y contrastada sobre la misma (Ziglio, 1996: 21).

3 Technology Road Mapping (hoja de ruta de evolución tecnológica, o mapas de evolución tecnológica): metodología en la que, en base a datos cuantitativos y cualitativos (básicamente entrevistas a expertos), se establece una secuencia lógica de desarrollo de una determinada tecnología, a corto, medio y largo plazo, estableciendo los principales factores que estimulan o retardan dicho proceso, y el conjunto de oportunidades y problemas que se derivan de su desarrollo.

4 La matriz de escenarios cruzados (MIC) es una técnica similar al método Delphi, pero que tiene en consideración tanto los pronósticos previsibles, como la interacción entre los mismos. "Una MIC describe dos tipos de datos para un conjunto de posibles desarrollos futuros: la probabilidad de que cada evento considerado ocurra en el período de tiempo especificado, y la probabilidad de ocurrencia de cada evento teniendo en cuenta la ocurrencia de cada uno de los restantes y el impacto estimado del segundo sobre el primero" (Bass, 1999: 127).

\section{BIBLIOGRAFÍA}

Abt, C. C. (2003): "El futuro de la energía desde la perspectiva de las ciencias sociales", en R. N. Cooper y R. Layard (eds.), ¿Qué nos depara el futuro? Perspectivas desde las ciencias sociales, Madrid, Alianza, pp. 87-138.

Bas, E. (1998): Prospectiva. Herramientas para la gestión estratégica del cambio, Barcelona, Ariel.

Castilla, A. (2000): "Cambios tecnológicos y escenarios del nuevo siglo", en J. F. Tezanos (ed.), Escenarios del nuevo siglo. Cuarto Foro sobre Tendencias Sociales, Madrid, Sistema, pp. 39-55.

Coates, J. F.; Mahaffie, J. B. y Hines, A. (1997): Scenarios of US and Global Society Reshaped by Science and Technology, Greensboro, Oakhill Press.

Collindridge, D. (1980): The Social Control of Technology, London, Pinter.

Estrategia Nacional de Ciencia y Tecnología (ENCYTE) (2008): Ejercicio de Prospectiva a 2020, Madrid, Fundación Española para la Ciencia y la Tecnología (FEYCT).

Eriksson, E. A. y Weber, K. M. (2008): "Adaptative Foresight: Navigating the complex landscape of policy strategies", Technological Forecasting and Social Change (doi:10.1016/ j.techfore.2008.02.06).

European Foundatioin for the Improvemente of Living and Working Conditions (EFILWC) (2003): Handobook of Knowledge Society Foresight, Dublin.

Fowles, J. (1978): The Handbook of Futures Research, Westport, CT, Greenwood Press.

Freeman, R. B. (2003): "El mundo del trabajo en el nuevo milenio", en R. N. Cooper y R. Layard (eds.), ¿Qué nos depara el futuro? Perspectivas desde las ciencias sociales, Madrid, Alianza, pp. 175-199.

Fuller, T.; Smedt, de P. y Rothman, D. S. (2006): Advancing Foresight Methodology through Networked Conversation, Second International Seville Seminar on Future-Oriented Techno- logy Analysis: Impact of FTA Approaches on Policy and Decision-Making, Seville 28-29 September (www.jrc. es).

Gabor, D. (1964): Inventing the Future, New York, Knopf.

Georgantzas, N. C. y Azcar, W. (1995): Scenario-Driven Planning, New York, Greenwood Publishing Group.

Georghiou, L.; Harper, J. C.; Keenan, M.; Miles, I. y Popper, R. (2008): The Handbook of Technology Foresight. Concepts and Practice, Cheltenham (UK), Edward Elgar Publishing.

Godet, M. (1991): Prospectiva y planificación estratégica, Barcelona, SG Editores.

Gries, W. H. (2000): "The CONVAIR Foresight Exercise in Information Communication Technology and Implications for the Foresight Process", in M. Häder, S. Häder (Hrsg.), Die DelphiTechnik in den sozialwissenschaften. Methodische Forschungen und innovative Anwendungen, Wiesbaden, Westdeutscher Verlag, pp. 20-28.

Häder, M. y Häder S. (Hrsg.) (2000): Die Delphi-Technik in den sozialwissenschaften. Methodische Forschungen und innovative Anwendungen, Wiesbaden, Westdeutscher Verlag.

Heidegger, M. (1975): "Die Zeit des Weltbildes", en Holzwege, Gesamtausgabe, vol. 5, Frankfurt, Vittorio Klosterman.

Helmer, 0. (1970): "Análisis político del futuro", en Revista Española de la Opinión Pública, n. ${ }^{\text {os } 21-22, ~ p p . ~ 79-88 . ~}$

Hernández de la Fuente, D. (2008): Oráculos griegos, Madrid, Alianza Editorial.

Jouvenel, de B. (1967): The Art of Conjeture, New York, Basic Books.

Kaplan, M. A. (1994): "Preface", in C. Sheffield, M. Alonso, M. A. Kaplan (eds.), The World of 2044: Technological development and the future of society, Minnesota, Paragon House, pp. 5-10. 
Keenan, M.; Butter, M.; Sainz de la Fuente, G. y Popper, R. (2006): Mapping foresight in Europe and other regions of the world. Highlights form the Annual mapping of the EFMN in 2005-2006, European Commission DG Research, Brussells.

Keenan, M.; Abbott, D.; Scapolo, F. y Zappacosta, M. (2003): Mapping Foresight Competence in Europe: The EUROFO$R E$ Pilot Project, IPTS Technical Report Series, EUR 20755 EN European Commission Joint Research Center, Seville, Spain.

Lamo de Espinosa, E.; González Garcia, J. M. y Torres Albero, C. (1995): La sociología del conocimiento y de la ciencia, Madrid, Alianza Editorial.

Landeta, J. (1999): El método Delphi. Una técnica de previsión para la incertidumbre, Barcelona, Ariel Practicum.

López Peláez, A. (2003): Nuevas tecnologías y sociedad actual: el impacto de la Robótica, Madrid, Instituto Nacional de Seguridad e Higiene en el Trabajo (MTAS).

López Peláez, A. y Kyriakou, D. (2008): "Robots, genes and bytes: technology development and social changes towards the year 2020", en Technological Forecasting and Social Change (doi: 10.1016/j.techfore.2008.01.002).

Ministére de I'Enseignement Supérieur et de la Recherche (MESR) (1995): En- quête sur les technologies du futur par la méthode Delphi, Paris.

Meadows, D. et al. (1972): The Limits of Growth, New York, New American Library.

Morato, A.; Rodríguez, A.; Miles, M.; Keenan, M.; Clar, G. y Svanfeldt, C. (2002): Guía práctica de prospectiva regional en España, Dirección General de Investigación, Comisión Europea, Luxemburgo.

Nacional Institute of Science and Technology Policy (NISTEP) (2005): Science and Technology Foresight Survey. Delphi Analysis, NISTEP Report n. ${ }^{\circ}$ 7, Tokyo (Japan), Ministry of Education, Culture, Sports, Science and Technology.

Observatorio de Prospectiva Tecnológica Industrial (OPTI) (1999): Primer Informe de Prospectiva Tecnológica Industrial. Futuro Tecnológico en el horizonte del 2015, Madrid, Ministerio de Industria y Energía.

Ringland, G.; Todd, K. y Schwartz, P. (1998): Scenario Planing: Mananging for the Future, New York, John Wiley \& Sons.

Scapolo, F.; Porter, A. L. y Rader, M. (2008): "Future-Oriented Technology Analysis (FTA): Impact on policy and decision making - The 2006 FTA International Seville Seminar", en Technological Forecasting and Social Change (doi: 10.1016 /j.techfore.2008.03.001).
Schwartz, P. (2003): "El río y la bola de billar: la historia, la innovación y el futuro", en R. N. Cooper y R. Layard (eds.), ¿Qué nos depara el futuro? Perspectivas desde las ciencias sociales, Madrid, Alianza, pp. 27-38.

Tezanos, J. F. (ed.) (2007): Los impactos sociales de la revolución científicotecnológica. Noveno Foro sobre Tendencias Sociales, Madrid, Sistema.

Tezanos, J. F. (ed.) (2008): Juventud y exclusión social. Décimo Foro sobre Tendencias Sociales, Madrid, Sistema.

Tezanos, J. F. y Villalón Ogayar, J. J. (2002): Estudio Delphi sobre Tendencias Económicas, Políticas y Sociales, Madrid, Sistema.

Villalón, J. J. (2007): "La expansión de Internet. Efectos socioestructurales previsibles en España", en J. F. Tezanos (ed.), Los impactos sociales de la revolución científico tecnológica. Noveno Foro sobre Tendencias Sociales, Madrid, Sistema, pp. 331354.

Wagar, W. W. (1991): The Next Three Futures, New York, Praeger Publishers.

Ziglio, E. (1996): "The Delphi method and its Contribution to Decision-Making", en M. Adler, E. Ziglio (eds.), Gazing into the Oracle. The Delphi Method and its Application to Social Policy and Public Health, Jessica kingsley Publishers, London, pp. 3-33. 